\title{
A Simple Theory of Asymmetric Linear Elasticity
}

\author{
Zehua Qiu \\ Institute of Crustal Dynamics, China Earthquake Administration, Beijing, China \\ Email: qzhbh@163.com
}

How to cite this paper: Qiu, Z.H. (2020) A Simple Theory of Asymmetric Linear Elasticity. World Journal of Mechanics, 10, 166-185. https://doi.org/10.4236/wjm.2020.1010012

Received: August 24, 2020

Accepted: October 10, 2020

Published: October 13, 2020

Copyright $\odot 2020$ by author(s) and Scientific Research Publishing Inc. This work is licensed under the Creative Commons Attribution International License (CC BY 4.0).

http://creativecommons.org/licenses/by/4.0/

\begin{abstract}
Rotation is antisymmetric and therefore is not a coherent element of the classical elastic theory, which is characterized by symmetry. A new theory of linear elasticity is developed from the concept of asymmetric strain, which is defined as the transpose of the deformation gradient tensor to involve rotation as well as symmetric strain. The new theory basically differs from the prevailing micropolar theory or couple stress theory in that it maintains the same basis as the classical theory of linear elasticity and does not need extra concepts, such as "microrotation" and "couple stresses". The constitutive relation of the new theory, the three-parameter Hooke's law, comes from the theorem about isotropic asymmetric linear elastic materials. Concise differential equations of translational motion are derived consequently giving the same velocity formula for P-wave and a different one for S-wave. Differential equations of rotational motion are derived with the introduction of spin, which has an intrinsic connection with rotation. According to the new theory, S-wave essentially has rotation as large as deviatoric strain and should be referred to as "shear wave" in the context of asymmetric strain. There are nine partial differential equations for the deformation harmony condition in the new theory; these are given with the first spatial differentiations of asymmetric strain. Formulas for rotation energy, in addition to those for (symmetric) strain energy, are derived to form a complete set of formulas for the total mechanical energy.
\end{abstract}

\section{Keywords}

Linear Elasticity, Asymmetric Linear Elasticity, Asymmetric Strain, Asymmetric Stress, Three-Parameter Hooke's Law

\section{Introduction}

Modern seismology is developed based on the classical theory of linear elasticity 
[1] [2] [3]. However, the emergence of "rotational seismology" [4] [5] [6] has presented another challenge to the classical elastic theory; that is, the theory may not be capable of solving problems regarding rotation, because "the angular motions are not coherently incorporated in it" [7].

On one hand, antisymmetric rotation has been observed with the use of various methods [8] [9] [10] [11] [12]. On the other hand, the prevailing classical elastic theory is characterized by the symmetry in the definitions of stress and strain, in Hooke's law, and, consequently, in the equations of motion. This mismatch has long been argued and addressed [7] [13] [14].

The logical conflict in modern seismological studies on rotation is very serious. Body waves, including P- and S-waves, are most commonly used concepts. Because P-wave is strictly defined as "irrotational wave" [1] [2], S-wave must have rotation; otherwise, it would not differ from $\mathrm{P}$-wave. The conflict lies in that S-wave, which should have rotation, is studied by modern seismology, which essentially excludes rotation.

The symmetry of the classical elastic theory initially came from Cauchy's second law of motion, which proved the symmetry of stress in 1827 [15]. The two fundamental principles in mechanics, namely, the momentum principle and the moment of momentum principle, can be applied to either a rigid body or a particle. Accordingly, a rigid body or a particle has six degrees of freedom in a Cartesian coordinate system: three corresponding to translation, and three to rotation or/and spin. Cauchy's second law of motion was achieved by applying the moment of momentum principle to an elastic body, which was regarded as a collection of particles, without considering spin. Spin is also antisymmetric and, as discussed later in this work, intrinsically connected with rotation.

In 1909, E. Cosserat and F. Cosserat introduced spin to develop a new theory of elasticity with asymmetric stress [13]. The seminal work of the Cosserat brothers, widely studied 50 years after it was published, has recently been commonly referred to as "micropolar theory" or "couple stress theory" [6] [7] [15]-[21]. The theory of micropolar elasticity additionally considers not only particle spin but also "microrotation" and "couple stresses," including surface couple and body couple stresses, to produce particle spin. For the micropolar continuum, there are six elastic constants, in contrast to the material of the classical elastic theory, for which there are only two. The theory of the Cosserat brothers has enlarged the scope of elasticity. However, its big leap from the classical elastic theory makes it difficult to apply to seismological studies [22].

In the present research, a new theory of asymmetric linear elasticity that is as simple as the classical symmetric theory is established through comparisons between the two in relation to seismology. The new theory basically differs from the prevailing micropolar theory or couple stress theory in that it has the same basis as the classical theory of linear elasticity and does not need extra concepts, such as "microrotation" and "couple stresses". The new theory is easy to understand as long as the concept of the spin of an infinitesimal block of deformable medium, but not of a rigid particle, is accepted. The new theory is an exact up- 
grade that incorporates rotation as a coherent part from the pure classical linear elastic theory, independent of nonlinearity, finite deformation, and source process. S-wave has a valid identity in the new theory, but its velocity formula has a different explanation.

\section{Stress and Strain}

A theory of linear elasticity studies only small deformation of ideal homogeneous elastic continuum, in which stress tensor and strain tensor are two fundamental concepts. In the classical linear elastic theory, stress and strain are symmetric; thus, they are here referred to as symmetric stress and symmetric strain, respectively. Correspondingly, in the new theory, stress and strain, which are asymmetric, are referred to as asymmetric stress and asymmetric strain, respectively.

In the Cartesian coordinate system $\left(x_{1}, x_{2}, x_{3}\right)$, symmetric stress is commonly given as

$$
\sigma_{i j}=\left[\begin{array}{lll}
\sigma_{11} & \sigma_{12} & \sigma_{13} \\
\sigma_{12} & \sigma_{22} & \sigma_{23} \\
\sigma_{13} & \sigma_{23} & \sigma_{33}
\end{array}\right],
$$

hence, $\sigma_{i j}=\sigma_{j i}(i, j=1,2,3)$. Meanwhile, given displacement $\boldsymbol{u}=\left(u_{1}, u_{2}, u_{3}\right)$, symmetric strain are defined by

$$
\varepsilon_{i j}=\left[\begin{array}{lll}
\varepsilon_{11} & \varepsilon_{12} & \varepsilon_{13} \\
\varepsilon_{12} & \varepsilon_{22} & \varepsilon_{23} \\
\varepsilon_{13} & \varepsilon_{23} & \varepsilon_{33}
\end{array}\right] \equiv \frac{1}{2}\left(u_{i, j}+u_{j, i}\right) \quad(i, j=1,2,3) ;
$$

therefore, $\varepsilon_{i j}=\varepsilon_{j i}$.

In contrast, asymmetric stress is given as

$$
p_{i j}=\left[\begin{array}{ccc}
p_{11} & p_{12} & p_{13} \\
p_{21} & p_{22} & p_{23} \\
p_{31} & p_{32} & p_{33}
\end{array}\right]
$$

and asymmetric strain is defined, in the new theory, by

$$
q_{i j}=\left[\begin{array}{lll}
q_{11} & q_{12} & q_{13} \\
q_{21} & q_{22} & q_{23} \\
q_{31} & q_{32} & q_{33}
\end{array}\right] \equiv u_{j, i}=\left[\begin{array}{lll}
\frac{\partial u_{1}}{\partial x_{1}} & \frac{\partial u_{2}}{\partial x_{1}} & \frac{\partial u_{3}}{\partial x_{1}} \\
\frac{\partial u_{1}}{\partial x_{2}} & \frac{\partial u_{2}}{\partial x_{2}} & \frac{\partial u_{3}}{\partial x_{2}} \\
\frac{\partial u_{1}}{\partial x_{3}} & \frac{\partial u_{2}}{\partial x_{3}} & \frac{\partial u_{3}}{\partial x_{3}}
\end{array}\right],
$$

where $p_{i j} \neq p_{j i}$ and $q_{i j} \neq q_{j i}(i \neq j)$, respectively.

It is important to note that in (4) the order of the subscripts of $u$ is " $j, i$ " and not " $i, j$ ". Only in this way do the shear strains match the corresponding shear stresses. As shown in Figure 1, for instance, $p_{21}$ and $p_{12}$ are different and have different effects on the infinitesimal block. They act in perpendicular directions and can produce unequal $\Delta u_{1} / \Delta x_{2} \approx q_{21}$ and $\Delta u_{2} / \Delta x_{1} \approx q_{12}$, respectively. 


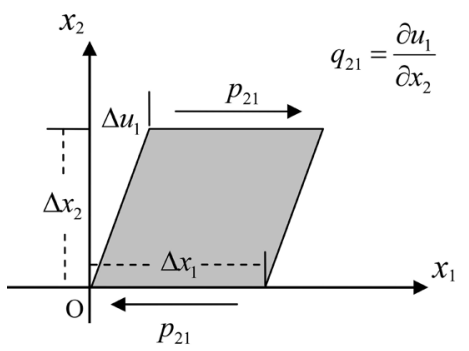

(a)

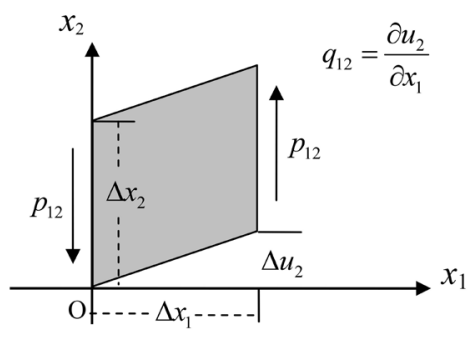

(b)

Figure 1. Diagram of the shear stresses and the corresponding shear strains in the $X_{1}-X_{2}$ plane.

The asymmetric strain tensor can always be uniquely divided into the symmetric strain tensor and an antisymmetric rotation tensor:

$$
q_{i j}=\frac{1}{2}\left(u_{j, i}+u_{i, j}\right)+\frac{1}{2}\left(u_{j, i}-u_{i, j}\right)=\varepsilon_{i j}+\omega_{i j},
$$

where rotation

$$
\omega_{i j}=\frac{1}{2}\left(u_{j, i}-u_{i, j}\right)=-\omega_{j i}=-\frac{1}{2}\left(u_{i, j}-u_{j, i}\right) .
$$

Rotation can also be written as a vector (Bullen, 1963, p. 14):

$$
\boldsymbol{\omega}=\left(\omega_{1}, \omega_{2}, \omega_{3}\right)=\frac{1}{2} \nabla \times \boldsymbol{u}=\left(\omega_{23}, \omega_{31}, \omega_{12}\right) .
$$

According to (7), rotation $\boldsymbol{\omega}$ is half the curl of displacement $\boldsymbol{u}$. Therefore, the term "rigid rotation" in the classical theory is misleading because the curl cannot be regarded as rigid unless it is spatially constant. A nonzero constant distribution of the displacement curl corresponds to the rotation of a finite rigid, not elastic, body around a fixed axis. In the case of elasticity, there is no room for such constant curl of displacement.

\section{Hooke's Law}

Hooke's law defines the linear relation between stress and strain. There are only six independent components in a symmetric stress or strain tensor; thus, the generalized Hooke's law in the classical theory can be given as

$$
\left[\begin{array}{l}
\sigma_{11} \\
\sigma_{12} \\
\sigma_{13} \\
\sigma_{22} \\
\sigma_{23} \\
\sigma_{33}
\end{array}\right]=\left[\begin{array}{llllll}
c_{11} & c_{12} & c_{13} & c_{14} & c_{15} & c_{16} \\
c_{21} & c_{22} & c_{23} & c_{24} & c_{25} & c_{26} \\
c_{31} & c_{32} & c_{33} & c_{34} & c_{35} & c_{36} \\
c_{41} & c_{42} & c_{43} & c_{44} & c_{45} & c_{46} \\
c_{51} & c_{52} & c_{53} & c_{54} & c_{55} & c_{56} \\
c_{61} & c_{62} & c_{63} & c_{64} & c_{65} & c_{66}
\end{array}\right]\left[\begin{array}{l}
\varepsilon_{11} \\
\varepsilon_{12} \\
\varepsilon_{13} \\
\varepsilon_{22} \\
\varepsilon_{23} \\
\varepsilon_{33}
\end{array}\right] .
$$

There are 36 elastic constants in this symmetric relation. When the symmetric medium is also isotropic, the number of constants is decreased to two, and the symmetric Hooke's law is obtained [23]:

$$
\sigma_{i j}=\lambda \theta \delta_{i j}+2 \mu \varepsilon_{i j} \text {, }
$$


where

$$
\theta=\nabla \cdot \boldsymbol{u}=\varepsilon_{k k}
$$

indicates dilatation, $\lambda$ and $\mu$ are Lamé's constants, and $\delta$ is the Kronecker delta. One remarkable but usually ignored point of the Hooke's law (9) is that it formally excludes rotation from the classical theory, which is effectively equivalent to assuming $\omega_{i j}=0$.

In the new asymmetric theory, the generalized Hooke's law is written as

$$
\left[\begin{array}{l}
p_{11} \\
p_{12} \\
p_{13} \\
p_{21} \\
p_{22} \\
p_{23} \\
p_{31} \\
p_{32} \\
p_{33}
\end{array}\right]=\left[\begin{array}{lllllllll}
c_{1111} & c_{1112} & c_{1113} & c_{1121} & c_{1122} & c_{1123} & c_{1131} & c_{1132} & c_{1133} \\
c_{1211} & c_{1212} & c_{1213} & c_{1221} & c_{1222} & c_{1223} & c_{1231} & c_{1232} & c_{1233} \\
c_{1311} & c_{1312} & c_{1313} & c_{1321} & c_{1322} & c_{1323} & c_{1331} & c_{1332} & c_{1333} \\
c_{2111} & c_{2112} & c_{2113} & c_{2121} & c_{2122} & c_{2123} & c_{2131} & c_{2132} & c_{2133} \\
c_{2211} & c_{2212} & c_{2213} & c_{2221} & c_{2222} & c_{2223} & c_{2231} & c_{2232} & c_{2233} \\
c_{2311} & c_{2312} & c_{2313} & c_{2321} & c_{2322} & c_{2323} & c_{2331} & c_{2332} & c_{2333} \\
c_{3111} & c_{3112} & c_{3113} & c_{3121} & c_{3122} & c_{3123} & c_{3131} & c_{3132} & c_{3133} \\
c_{3211} & c_{3212} & c_{3213} & c_{3221} & c_{3222} & c_{3223} & c_{3231} & c_{3232} & c_{3233} \\
c_{3311} & c_{3312} & c_{3313} & c_{3321} & c_{3322} & c_{3323} & c_{3331} & c_{3332} & c_{3333}
\end{array}\right]\left[\begin{array}{l}
q_{11} \\
q_{12} \\
q_{13} \\
q_{21} \\
q_{22} \\
q_{23} \\
q_{31} \\
q_{32} \\
q_{33}
\end{array}\right],
$$

where the 81 constants produces an entire fourth-order tensor $c_{i j m n}$ $(i, j, m, n=1,2,3)$. Relation (11) can be concisely rewritten as

$$
p_{i j}=c_{i j m n} q_{m n} .
$$

When the asymmetric medium is isotropic, the number of independent elastic constants is decreased to three, and the asymmetric Hooke's law can be written as [24]:

$$
p_{i j}=\lambda \delta_{i j} \theta+\mu_{1} q_{i j}+\mu_{2} q_{j i}
$$

or

$$
p_{i j}=\lambda \delta_{i j} \theta+\left(\mu_{1}+\mu_{2}\right) \varepsilon_{i j}+\left(\mu_{1}-\mu_{2}\right) \omega_{i j} .
$$

where

$$
\theta=\nabla \cdot \boldsymbol{u}=q_{k k}=\varepsilon_{k k} .
$$

$\mu_{1}$ and $\mu_{2}$ are two independent constants, here termed the parallel and the perpendicular shear modulus, respectively. When there is symmetry, $p_{i j}$ becomes $\sigma_{i j}$ and $\left(\mu_{1}-\mu_{2}\right) \omega_{i j}=0$; hence, relation (14) converts back to (9), and

$$
\mu=\left(\mu_{1}+\mu_{2}\right) / 2 \text {. }
$$

The asymmetric stress tensor can be further divided into the symmetric stress tensor and an antisymmetric tensor $\eta_{i j}$ :

$$
p_{i j}=\left(p_{i j}+p_{j i}\right) / 2+\left(p_{i j}-p_{j i}\right) / 2=\sigma_{i j}+\eta_{i j} .
$$

Here, $\eta_{i j}=\left(p_{i j}-p_{j i}\right) / 2$ is termed stress torque, which can also be written as a vector. By introducing (9) into (17), which is then compared with (14), the cause of rotation is eventually found:

$$
\eta_{i j}=\left(\mu_{1}-\mu_{2}\right) \omega_{i j} \quad(i \neq j) \text { or } \eta=\left(\mu_{1}-\mu_{2}\right) \omega .
$$


Equation (18) implies that rotation be caused by stress torque. The reverse is also reasonable; i.e., stress torque is induced by rotation.

Equation (18) is important because it reveals the existence of stress asymmetry, which is determined by two factors, $\mu_{1} \neq \mu_{2}$ and $\omega_{i j} \neq 0$; i.e., the medium must be asymmetric, and there must be rotation.

Regarding the theory of elasticity, the medium is physically more important in determining the property of the wave. From the fact that S-waves are always observed in seismic recordings, it can be inferred that earth materials are generally asymmetric, where $\mu_{1} \neq \mu_{2}$. When $\mu_{1}=\mu_{2}$, (14) reverts to (9), and an asymmetric medium turns back to a symmetric medium. In a symmetric medium, even if rotation is produced at a source, it will not be able to spread out.

Other elastic constants for the new asymmetric theory can be easily rewritten with the substitution in (16). For example:

$$
\text { Young's modulus } E=\frac{\mu(3 \lambda+2 \mu)}{\lambda+\mu}=\frac{\left(\mu_{1}+\mu_{2}\right)\left(3 \lambda+\mu_{1}+\mu_{2}\right)}{2 \lambda+\mu_{1}+\mu_{2}}
$$

and

$$
\text { Poisson's ratio } v=\frac{\lambda}{2(\lambda+\mu)}=\frac{\lambda}{2 \lambda+\mu_{1}+\mu_{2}} \text {. }
$$

\section{Differential Equations of Translational Motion}

Differential equations of translational motion are originally derived from the momentum principle or Newton's second law. In seismology, the term of body force in the equations is often ignored to obtain the equations of body waves.

In the classical theory of linear elasticity, the differential equation of translational motion in terms of stress can be written in indicial notation as

$$
\rho \frac{\partial^{2} u_{j}}{\partial t^{2}}=\sigma_{i j, i} \quad(i, j=1,2,3)
$$

where $\rho$ indicates density. By introducing the symmetric Hooke's law (9), stress is replaced with strain, and the equation becomes

$$
\rho \frac{\partial^{2} u_{j}}{\partial t^{2}}=\lambda \frac{\partial \theta}{\partial x_{j}}+2 \mu \varepsilon_{i j, i} \quad(i, j=1,2,3) .
$$

Further plugging (2) into (22) for displacement to substitute for strain finally obtains the equation in vector notation [23]:

$$
\rho \frac{\partial^{2} \boldsymbol{u}}{\partial t^{2}}=(\lambda+\mu) \nabla \theta+\mu \nabla^{2} \boldsymbol{u}
$$

Equation (23) can be rewritten by using the general relation

$$
\nabla^{2} \boldsymbol{u}=\nabla \theta-2 \nabla \times \boldsymbol{\omega}
$$

as

$$
\rho \frac{\partial^{2} \boldsymbol{u}}{\partial t^{2}}=(\lambda+2 \mu) \nabla^{2} \boldsymbol{u}+2(\lambda+\mu) \nabla \times \boldsymbol{\omega}
$$


When rotation is absent, $\nabla \times \omega \equiv 0$, the following equation for P-wave is obtained from (25):

$$
\frac{\partial^{2} \boldsymbol{u}}{\partial t^{2}}=\frac{\lambda+2 \mu}{\rho} \nabla^{2} \boldsymbol{u}
$$

which yields P-wave velocity as

$$
\alpha^{\prime}=\sqrt{\frac{\lambda+2 \mu}{\rho}} .
$$

This is why P-wave is termed "irrotational wave" [1] [2].

In the classical theory, when there is no change in dilatation, $\nabla \theta \equiv 0,(23)$ is changed into the equation for S-wave

$$
\frac{\partial^{2} \boldsymbol{u}}{\partial t^{2}}=\frac{\mu}{\rho} \nabla^{2} \boldsymbol{u}
$$

and S-wave velocity is expressed as

$$
\beta^{\prime}=\sqrt{\frac{\mu}{\rho}}
$$

This is why S-wave is called "equivoluminal wave" [2].

However, (28) and (29) may not be valid. As concluded in the previous chapter, $\omega_{i j}=0$ is implicitly assumed in the establishment of the symmetric Hooke's law (9) in the classical theory. Therefore, the relation (24) becomes

$$
\nabla^{2} \boldsymbol{u}=\nabla \theta .
$$

By substituting (30) into (23), Equation (26) is again obtained, but not (28). This implies that there is only one type of body wave, namely, P-wave, which propagates in a symmetric medium.

In the new asymmetric theory, corresponding to the translational Equations (21)-(23), we obtain by a similar derivation the equation in terms of stress

$$
\rho \frac{\partial^{2} u_{j}}{\partial t^{2}}=p_{i j, i} \quad(i, j=1,2,3)
$$

then, by applying the asymmetric Hooke's law (13), the equation in terms of strain

$$
\rho \frac{\partial^{2} u_{j}}{\partial t^{2}}=\lambda \frac{\partial \theta}{\partial x_{j}}+\mu_{1} q_{i j, i}+\mu_{2} q_{j i, i} \quad(i, j=1,2,3),
$$

and, by further applying (4), the equation in terms of displacement

$$
\rho \frac{\partial^{2} \boldsymbol{u}}{\partial t^{2}}=\left(\lambda+\mu_{2}\right) \nabla \theta+\mu_{1} \nabla^{2} \boldsymbol{u} .
$$

By using the general relation (24), Equation (33) can be rewritten as

$$
\rho \frac{\partial^{2} \boldsymbol{u}}{\partial t^{2}}=\left(\lambda+\mu_{1}+\mu_{2}\right) \nabla^{2} \boldsymbol{u}+2\left(\lambda+\mu_{2}\right) \nabla \times \omega .
$$

For "irrotational" P-wave, $\nabla \times \omega \equiv 0$; thus, from (34) are obtained P-wave equation 


$$
\frac{\partial^{2} \boldsymbol{u}}{\partial t^{2}}=\frac{\lambda+\mu_{1}+\mu_{2}}{\rho} \nabla^{2} \boldsymbol{u}
$$

and the formula for P-wave velocity

$$
\alpha=\sqrt{\frac{\lambda+\mu_{1}+\mu_{2}}{\rho}} .
$$

Equation (36) is in fact the same as (27).

Regarding "equivoluminal" S-wave, $\nabla \theta \equiv 0$; thus from (33) are obtained S-wave equation

$$
\frac{\partial^{2} \boldsymbol{u}}{\partial t^{2}}=\frac{\mu_{1}}{\rho} \nabla^{2} \boldsymbol{u}
$$

and the formula for S-wave velocity

$$
\beta=\sqrt{\frac{\mu_{1}}{\rho}} .
$$

Interestingly, S-wave velocity is determined only by $\mu_{1}$ but without $\mu_{2}$. Equation (38) is similar to, but factually different from, (29) because $\mu \neq \mu_{1}$. Physically, $\mu_{1}>\mu_{2}$ in real materials. Therefore, because $2 \mu=\mu_{1}+\mu_{2}$, then $\mu_{1}>\mu$.

The velocity formulas for $\mathrm{P}$ - and $\mathrm{S}$-waves can also be derived by introducing a scalar potential $\phi(x, t)$ and a vector potential $\boldsymbol{\psi}(\boldsymbol{x}, t)$ to separate the displacement field into two exclusively different parts, one with no rotation and the other with no dilatation [23]:

$$
\boldsymbol{u}=\nabla \phi(\boldsymbol{x}, t)+\nabla \times \boldsymbol{\psi}(\boldsymbol{x}, t) .
$$

This can be proven to achieve the same results (see Appendix). Equation (39) clarifies that there are only two kinds of body waves, $\mathrm{P}$-wave corresponding to $\phi(x, t)$ and S-wave corresponding to $\psi(x, t)$. Logically, the derivation of the classical theory should refer to only the part of the displacement associated with $\phi(x, t)$ because it essentially excludes rotation.

\section{Deformation Constitution of P- and S-Waves}

The entire asymmetric strain tensor can be divided into three portions, including the mean strain tensor $\theta \delta_{i j} / 3$, the strain deviator tensor $\varepsilon_{i j}^{D}$, and the rotation tensor:

$$
q_{i j}=\theta \delta_{i j} / 3+\varepsilon_{i j}^{D}+\omega_{i j}
$$

where the strain deviator

$$
\varepsilon_{i j}^{D}=\varepsilon_{i j}-\theta \delta_{i j} / 3 .
$$

P- and S-waves are also reasonably called "dilatational" and "rotational" waves, respectively [2]. Interestingly, according to (39), whereas dilatation must go with $\mathrm{P}$-wave, and rotation with $\mathrm{S}$-wave, deviatoric strain is left to be explained.

Based on the above discussions, $\mathrm{P}$-wave has dilatation, and $\mathrm{S}$-wave has rotation. However, it is not that merely dilatation travels with $\mathrm{P}$-wave and rotation alone 
goes with S-wave. According to the condition of compatibility to be discussed later, neither rotation nor dilatation can propagate by itself. In fact, the deviatoric strain has to be divided into two parts, one with dilatation in $\mathrm{P}$-wave and the other with rotation in S-wave.

Rewrite (39) as

$$
\boldsymbol{u}=\boldsymbol{u}^{P}+\boldsymbol{u}^{S}
$$

where $\boldsymbol{u}^{P}=\nabla \phi(\boldsymbol{x}, t)$ and $\boldsymbol{u}^{S}=\nabla \times \boldsymbol{\psi}(\boldsymbol{x}, t)$; therefore,

$$
q_{i j}=q_{i j}^{P}+q_{i j}^{S}=u_{j, i}^{P}+u_{j, i}^{S}
$$

Figure 2 shows how, in the $\left(x_{1}, x_{2}\right)$ plane, elastic material deforms in $\mathrm{P}$-wave (longitudinal wave) and S-wave (transverse wave), respectively, which proceed in the $x_{1}$ direction as plane waves. For $\mathrm{P}$-wave, the strain tensor is

$$
q_{i j}^{p}=\left[\begin{array}{ccc}
u_{1,1} & 0 & 0 \\
0 & 0 & 0 \\
0 & 0 & 0
\end{array}\right],
$$

where $\theta=u_{1,1}$. For S-wave, the strain tensor is

$$
q_{i j}^{S}=\left[\begin{array}{ccc}
0 & u_{2,1} & 0 \\
0 & 0 & 0 \\
0 & 0 & 0
\end{array}\right] .
$$

Note that (45) is an asymmetric tensor and $\theta=0$. This is the simplest expression of S-wave to prove its asymmetry.

The symmetric strain of P-wave involves two parts: mean strain and deviatoric strain:

$$
q_{i j}^{p}=\frac{1}{3}\left[\begin{array}{ccc}
u_{1,1} & 0 & 0 \\
0 & u_{1,1} & 0 \\
0 & 0 & u_{1,1}
\end{array}\right]+\frac{1}{3}\left[\begin{array}{ccc}
2 u_{1,1} & 0 & 0 \\
0 & -u_{1,1} & 0 \\
0 & 0 & -u_{1,1}
\end{array}\right] \text {, }
$$
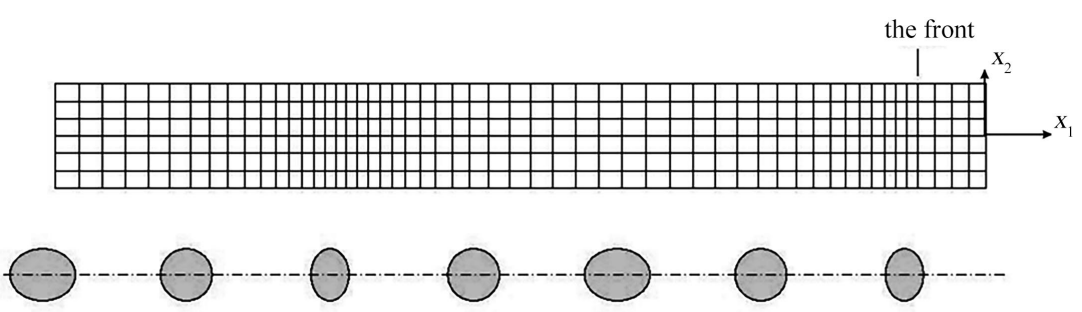

(a)

the front
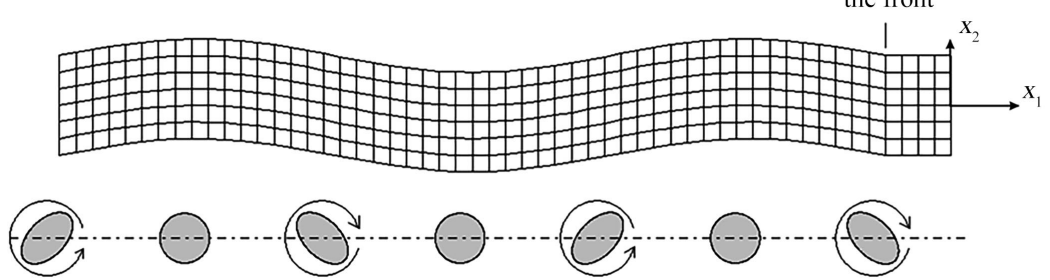

(b)

Figure 2. Diagrams of the deformations of P-wave (a), which has only symmetric strain, and S-wave (b), which has both symmetric strain and rotation. 
As shown in Figure 2(a), the material deforms in the way that circles and ovals alternate with each other in P-wave. Physically, there is shearing when a circle deforms into an oval and vice versa.

The asymmetric strain of S-wave also contains two parts: deviatoric strain and rotation:

$$
q_{i j}^{S}=\frac{1}{2}\left[\begin{array}{ccc}
0 & u_{2,1} & 0 \\
u_{2,1} & 0 & 0 \\
0 & 0 & 0
\end{array}\right]+\frac{1}{2}\left[\begin{array}{ccc}
0 & u_{2,1} & 0 \\
-u_{2,1} & 0 & 0 \\
0 & 0 & 0
\end{array}\right] .
$$

As shown in Figure 2(b), rotation must be considered together with deviatoric strain for S-wave. Note that, according to (47), the amount of rotation is not smaller than but just as large as that of the deviatoric strain in S-wave. In fact, rotation and deviatoric strain occur as one inseparable identity in S-wave, as indicated by (45).

In general, the real deformation constitution of a certain wave should satisfy the condition of compatibility. This requires that continuous single-valued displacements can be obtained by integrating the strains.

In the classical theory, there are six strain-displacement relations given by (2), which are functions of three displacements. The compatibility condition is given with the second differentiations of $\varepsilon_{i j}$ with respect to $x_{i}$ [1] [25] [26] [27].

In the new theory, from the strain-displacement relations given by (4), the equations of the compatibility condition can be derived in a similar way with only the first differentiations of $q_{i j}$ with respect to $x_{i}$, such as

$$
\left\{\begin{array}{l}
\frac{\partial q_{11}}{\partial x_{2}}\left(=\frac{\partial^{2} u_{1}}{\partial x_{1} \partial x_{2}}\right)=\frac{\partial q_{21}}{\partial x_{1}}\left(=\frac{\partial^{2} u_{1}}{\partial x_{2} \partial x_{1}}\right) \\
\frac{\partial q_{21}}{\partial x_{3}}\left(=\frac{\partial^{2} u_{1}}{\partial x_{2} \partial x_{3}}\right)=\frac{\partial q_{31}}{\partial x_{2}}\left(=\frac{\partial^{2} u_{1}}{\partial x_{3} \partial x_{2}}\right) \\
\frac{\partial q_{31}}{\partial x_{1}}\left(=\frac{\partial^{2} u_{1}}{\partial x_{3} \partial x_{1}}\right)=\frac{\partial q_{11}}{\partial x_{3}}\left(=\frac{\partial^{2} u_{1}}{\partial x_{1} \partial x_{3}}\right)
\end{array}\right.
$$

In three dimensions, a total of nine equations of the compatibility condition are obtained for the asymmetric strain field to exist:

$$
\left(\begin{array}{llll}
\frac{\partial q_{11}}{\partial x_{2}}=\frac{\partial q_{21}}{\partial x_{1}} & \frac{\partial q_{12}}{\partial x_{2}}=\frac{\partial q_{22}}{\partial x_{1}} & \frac{\partial q_{13}}{\partial x_{2}}=\frac{\partial q_{23}}{\partial x_{1}} \\
\frac{\partial q_{21}}{\partial x_{3}}=\frac{\partial q_{31}}{\partial x_{2}} & \frac{\partial q_{22}}{\partial x_{3}}=\frac{\partial q_{32}}{\partial x_{2}} & \frac{\partial q_{23}}{\partial x_{3}}=\frac{\partial q_{33}}{\partial x_{2}} \\
\frac{\partial q_{31}}{\partial x_{1}}=\frac{\partial q_{11}}{\partial x_{3}} & \frac{\partial q_{32}}{\partial x_{1}}=\frac{\partial q_{12}}{\partial x_{3}} & \frac{\partial q_{33}}{\partial x_{1}}=\frac{\partial q_{13}}{\partial x_{3}}
\end{array}\right)
$$

or

$$
q_{i j, k}=q_{k j, i} .
$$

Assuming that the $x_{1}$-axis directs the proceeding of the wave so that $q_{i j, 1} \neq 0$, it can be easily concluded from (49) that mean strain (dilatation) 


$$
\theta \delta_{i j} / 3=\left[\begin{array}{ccc}
\theta / 3 & 0 & 0 \\
0 & \theta / 3 & 0 \\
0 & 0 & \theta / 3
\end{array}\right]
$$

cannot propagate by itself because it does not meet the conditions of $q_{12,2}(=0)=q_{22,1}(\neq 0)$ and/or $q_{33,1}(\neq 0)=q_{13,3}(=0)$. Similarly, rotation

$$
\omega_{i j}=\left[\begin{array}{ccc}
0 & \omega_{3} & -\omega_{2} \\
-\omega_{3} & 0 & \omega_{1} \\
\omega_{2} & -\omega_{1} & 0
\end{array}\right]
$$

alone cannot propagate because it does not satisfy $q_{11,2}(=0)=q_{21,1}(\neq 0)$ and/or $q_{31,1}(\neq 0)=q_{11,3}(=0)$. P-waves related to (44) can propagate because it meets the conditions $q_{11,2}(=0)=q_{21,1}(=0)$ and $q_{31,1}(=0)=q_{11,3}(=0)$. S-wave related to (45) can propagate because it meets the conditions $q_{12,2}(=0)=q_{22,1}(=0)$ and $q_{32,1}(=0)=q_{12,3}(=0)$.

\section{Differential Equations of Rotational Motion}

Differential equations of rotational motion are originally derived from the moment of momentum principle. In the classical theory, there is no such equation because there is no rotation. Instead, the symmetry of stress occurs in the static equilibrium of the moment of momentum [23]. In the new asymmetric theory, the differential equations can be easily deduced by comparison of the integral equation of the theory of micropolar elasticity with that of the new theory regarding the moment of momentum principle.

In the classical theory, spin is not considered when the moment of momentum principle is applied to a body of volume $\mathrm{V}$ and surface $\mathrm{S}$ to establish the following equation in the integral form [3] [15] [25]:

$$
\frac{\mathrm{d}}{\mathrm{d} t} \int_{V} \boldsymbol{r} \times \rho \boldsymbol{v} \mathrm{d} V=\int_{S} \boldsymbol{r} \times \boldsymbol{t} \mathrm{d} S+\int_{V} \boldsymbol{r} \times \rho \boldsymbol{b} \mathrm{d} V,
$$

where $\boldsymbol{v}=\partial \boldsymbol{u} / \partial t, \boldsymbol{r}=\boldsymbol{x}-\boldsymbol{x}_{0}, \boldsymbol{x}_{0}$ denotes the position of the reference point of the moments, $\boldsymbol{b}$ represents the body force, and the surface traction $\boldsymbol{t}$ can be written in indicial notation as

$$
t_{j}=p_{i j} n_{i},
$$

where $n_{i}$ is the normal vector of S. The term on the left-hand side of (53) includes the angular momentums of all parts of the body about the reference point, but not spin. The integral Equation (53) results in the symmetry of stress [3] [15] [25]:

$$
p_{i j}-p_{j i}=0 \text { or } \sigma_{i j}=\sigma_{j i}(i \neq j) .
$$

Equation (55) is referred to as Cauchy's second law of motion [15] and has been followed ever since by the classical theory.

When spin is additionally incorporated, the following integral equation for the new theory is obtained: 


$$
\frac{\mathrm{d}}{\mathrm{d} t} \int_{V}(\boldsymbol{r} \times \rho \boldsymbol{v}+\rho \boldsymbol{s}) \mathrm{d} V=\int_{S} \boldsymbol{r} \times \boldsymbol{t} \mathrm{d} S+\int_{V} \boldsymbol{r} \times \rho \boldsymbol{b} \mathrm{d} V .
$$

where $s$ stands for spin, that is, the spin angular momentum per unit mass of an infinitesimal volume of the body. In order to deduce the differential equation of rotational motion from Equation (56), the integral equation of angular momentum of the theory of micropolar elasticity is first considered [15] [17] [21].

In the theory of micropolar elasticity, spin can be produced by couple stresses, including surface couples and body couples. The integral equation of the theory of micropolar elasticity takes the form [15]

$$
\frac{\mathrm{d}}{\mathrm{d} t} \int_{V}(\boldsymbol{r} \times \rho \boldsymbol{v}+\rho \boldsymbol{s}) \mathrm{d} V=\int_{S}(\boldsymbol{r} \times \boldsymbol{t}+\boldsymbol{m}) \mathrm{d} S+\int_{V}(\boldsymbol{r} \times \rho \boldsymbol{b}+\rho \boldsymbol{c}) \mathrm{d} V,
$$

where $\boldsymbol{m}$ and $\boldsymbol{c}$ are, respectively, the surface and body couples to produce spin. After a difficult derivation, an inference from (57) takes the form [15]

$$
\frac{\partial M_{l i}}{\partial x_{l}}+\rho c_{i}+e_{i j k} p_{j k}=\rho \frac{\partial s_{i}}{\partial t}
$$

where $M_{l i}$ is defined by $m_{i}=M_{l i} n_{l}$, and $e_{i j k}$ is the permutation symbol. Note that the term $e_{i j k} p_{j k}$ may produce $\rho \partial s_{i} / \partial t$ as well as the couple stresses in the left-hand expression of (58).

A comparison of (56) with (57) shows that the former can be regarded as a shortened form of the latter by removing the couple stresses. In the new theory of asymmetric linear elasticity, couple stresses do not need to be introduced as the cause of spin. By canceling the terms of couples in (58), the differential equation of rotational motion of the new theory can be straightforwardly obtained:

$$
e_{i j k} p_{j k}=\rho \frac{\partial s_{i}}{\partial t} \text {. }
$$

Equation (59) can be expanded as

$$
\begin{aligned}
& i=1, \quad p_{23}-p_{32}=\rho \partial s_{1} / \partial t \\
& i=2, \quad p_{31}-p_{13}=\rho \partial s_{2} / \partial t \\
& i=3, \quad p_{12}-p_{21}=\rho \partial s_{3} / \partial t
\end{aligned}
$$

or written in vector notation:

$$
2 \eta=\rho \frac{\partial s}{\partial t}
$$

Equation (61) shows that spin can be caused by stress torque or, put the other way around, stress torque is induced by spin.

By comparing (18) and (61), the intrinsic connection between rotation and spin can be easily determined:

$$
\frac{1}{2} \rho \frac{\partial \mathbf{s}}{\partial t}=\left(\mu_{1}-\mu_{2}\right) \omega
$$

The time-dependent property of $\boldsymbol{s}$, which is a quantity of motion, indicates that rotation should be related only to dynamic problems and does not exist stat- 
ically. Thus, we clarify that without rotation, the theory of symmetric linear elasticity can be perfectly valid in the study of static problems.

Note that spin is here in the new theory referred to an infinitesimal block of deformable medium, but not to a rigid particle as in the theory of micropolar elasticity. The former can bear shear stress, whereas the latter cannot.

\section{Rotation Energy}

For simplicity, in the following discussion, the term "strain" is used for symmetric strain, and "deformation" for asymmetric strain. Accordingly, strain energy refers to symmetric strain energy, and deformation energy denotes asymmetric strain energy, which additionally involves rotation energy.

Disregarding body force and dissipation, the total mechanical energy $U$ of an elastic body is equal to the sum of the work done by the surface traction:

$$
U=\frac{1}{2} \int_{S} t_{i} u_{i} \mathrm{~d} S .
$$

In the symmetric theory, the surface traction

$$
t_{i}=\sigma_{i j} n_{j}
$$

Applying (64) to (63) results in

$$
\begin{aligned}
U^{\prime} & =\frac{1}{2} \int_{S} \sigma_{j i} n_{j} u_{i} \mathrm{~d} S \\
& =\frac{1}{2} \int_{V}\left(\sigma_{j i} u_{i}\right)_{, j} \mathrm{~d} V \\
& =\frac{1}{2} \int_{V}\left(\sigma_{j i, j} u_{i}+\sigma_{j i} u_{i, j}\right) \mathrm{d} V \\
& =\frac{1}{2} \int_{V}\left(\sigma_{j i, j} u_{i}+\sigma_{j i} \varepsilon_{j i}\right) \mathrm{d} V
\end{aligned}
$$

Based on (65), the total mechanical energy $U^{\prime}$ consists of two parts: the energy associated with motion (or kinetic energy) and strain energy. According to Equation (21), kinetic energy

$$
U_{K}^{\prime}=\frac{1}{2} \int_{V} \sigma_{j i, j} u_{i} \mathrm{~d} V=\frac{1}{2} \rho \int_{V} \frac{\partial^{2} u_{i}}{\partial t^{2}} u_{i} \mathrm{~d} V,
$$

and strain energy (e. g., Stein \& Wysession, 2003)

$$
U_{S}=\frac{1}{2} \int_{V} \sigma_{i j} \varepsilon_{i j} \mathrm{~d} V .
$$

In the new theory, the surface traction

$$
t_{i}=p_{j i} n_{j} .
$$

Accordingly, by a similar derivation to (65), the total mechanical energy is obtained as

$$
U=\frac{1}{2} \int_{V}\left(p_{j i, j} u_{i}+p_{j i} q_{j i}\right) \mathrm{d} V,
$$


which also consists of two parts: kinetic energy

$$
U_{K}=\frac{1}{2} \int_{V} p_{j i, j} u_{i} \mathrm{~d} V=\frac{1}{2} \int_{V}\left(\sigma_{j i, j} u_{i}+\eta_{j i, j} u_{i}\right) \mathrm{d} V,
$$

and deformation energy

$$
\begin{aligned}
U_{D} & =\frac{1}{2} \int_{V} p_{i j} q_{i j} \mathrm{~d} V \\
& =\frac{1}{2} \int_{V}\left(\sigma_{i j}+\eta_{i j}\right)\left(\varepsilon_{i j}+\omega_{i j}\right) \mathrm{d} V \\
& =\frac{1}{2} \int_{V}\left(\sigma_{i j} \varepsilon_{i j}+\sigma_{i j} \omega_{i j}+\eta_{i j} \varepsilon_{i j}+\eta_{i j} \omega_{i j}\right) \mathrm{d} V \\
& =\frac{1}{2} \int_{V}\left(\sigma_{i j} \varepsilon_{i j}+\eta_{i j} \omega_{i j}\right) \mathrm{d} V
\end{aligned}
$$

Note that $\sigma_{i j} \omega_{i j}=0$ and $\eta_{i j} \varepsilon_{i j}=0$. Therefore, rotation energy, i.e., the energy associated with rotation, is given in the form of a volume integral as

$$
U^{R}=U-U^{\prime}=\frac{1}{2} \int_{V}\left(\eta_{j i, j} u_{i}+\eta_{i j} \omega_{i j}\right) \mathrm{d} V
$$

Rotation energy can also be written in the form of a surface integral as

$$
U^{R}=\frac{1}{2} \int_{S}\left(p_{i j}-\sigma_{i j}\right) n_{j} u_{i} \mathrm{~d} S=\frac{1}{2} \int_{S} \eta_{i j} n_{j} u_{i} \mathrm{~d} S
$$

The term "rigid rotation" may mislead one into thinking that there is no deformation energy associated with rotation. In fact, however, there is. Deformation should not be related merely to an infinitesimal block itself. Deformation occurs due to the spatial variation of displacement or, in other words, due to deformational interactions between adjacent infinitesimal blocks. Figure 3 shows a deformational interaction between an infinitesimal area and its neighborhood, in which either strain or rotation of the infinitesimal area appears. When strain occurs to the imaginary circle, it becomes an oval. However, only such transformation of the area shape is not the real meaning of deformation. What's important is that its neighborhood is affected to deform. When rotation occurs to the circle, although the circle itself does not seem to deform, the neighborhood is affected to deform, too.

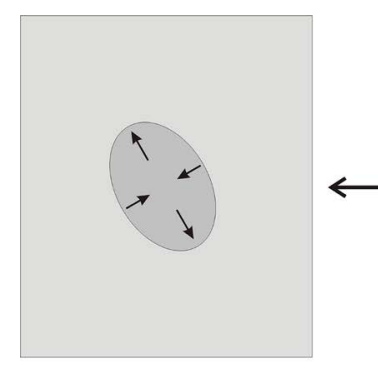

Strain
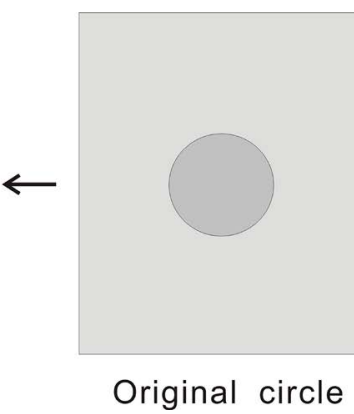

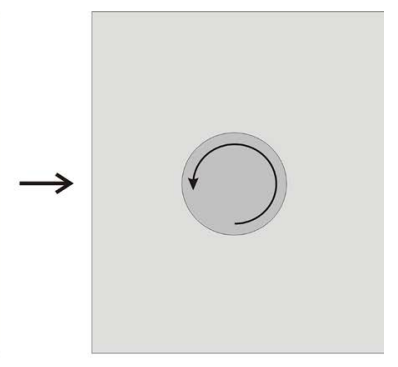

Rotation

Figure 3. Schematic illustration of deformation induced by strain and rotation, respectively. 


\section{Discussion}

The fact that rotation is not incorporated in the classical theory has already been recognized by many researchers but may still be unclear to others. Some consider rotation to be implicitly involved in, but not excluded from, the equations of the classical theory, a view that is wrong because it is impossible. Mathematically, when rotation is considered, it appears as nonzero. Otherwise, it is zero and hence not involved. In fact, rotation is treated inconsistently in the classical theory, being excluded from the core but included in the inferences, for example, on the S-wave velocity.

Given the study results on the fourth-order tensor, it is not difficult to figure out the asymmetric Hooke's law (13), which results in the definition of stress torque and its relation to rotation (18). Also, given the equations of rotational motion of the theory of micropolar elasticity (57) and (58), it is easy to draw the conclusion of the new theory regarding the relation between stress torque and spin (61). Both relations in (18) and (61) confirm the asymmetry of stress and the association between rotation and spin.

The focus is on rotation, which is essentially excluded from the core of the classical theory. The prevailing asymmetric theories that address this problem originated from the Cosserat brothers' theory, which uses the concept of particle spin, followed by couple stresses, to explain rotation. However, particle spin is not an appropriate counterpart. The rotation involved is initially defined in terms of a deformation gradient; thus, the problem must be tackled by using relevant concepts. The introduction of the concept of particle spin is misleading and renders the asymmetric theories too sophisticated to be applicable.

The introduction of spin adds three more degrees of freedom to the infinitesimal block in the classical theory to make up a total of six, as required by Newton's laws of mechanics. However, the relation (62) links spin and rotation together to reduce the degrees of freedom from six to three. Therefore, solutions of the problems in the new theory can also be given in terms of only displacements, without spin.

Because S-wave is intrinsically rotational, there would be no S-wave without rotation. Factually, however, S-waves are always observed in seismic recordings, so there must be rotation although usually not directly observable due to the lack of proper instruments.

The new theory has shown for the first time the deformation constitution of S-wave (45) and has clarified that when S-wave is called "shear wave," it should be in the context of asymmetric strain, which includes both symmetric strain and rotation (47). Moreover, according to (47), rotation is not as small as "negligible" [28] but generally is as large as the deviatoric strain in S waves.

\section{Conclusions}

The new theory of asymmetric linear elasticity is established self-consistently in logic. It provides a better description of the mechanical behavior of the ideal me- 
dium of linear elasticity. Some main points are listed as follows:

- The constitutive relation of the new theory, the three-parameter Hooke's law (13), is established based on the definition of asymmetric strain (4) and the theorem about isotropic asymmetric linear elastic materials. The new Hooke's law (13) reveals that rotation is caused by stress torque, the difference between unequal conjugate shear stresses.

- Concise differential equations of translational motion are derived consequently giving the same velocity formula for P-wave and a different one for S-wave (38). For instance, the new formula for S-wave velocity provides the key to solving the problem of why the "static moduli" of rocks are usually significantly smaller than the "dynamic moduli" [29] [30]. More importantly, the prevailing earth model has to be modified for those elastic parameters calculated with the old formulas of S-wave velocity.

- Differential equations of rotational motion are developed with the introduction of spin, which has an intrinsic connection with rotation. It further unveils the relation between rotation and stress torque.

- There are nine partial differential equations for the deformation harmony condition in the new theory; these are given with the first spatial differentiations of the asymmetric strain. For instance, according to the new condition, S-wave cannot travel without either symmetric shear strain or rotation. Similarly, some theoretical conclusions in modern seismology should be reexamined with this condition.

- Formulas for rotation energy, in addition to those for strain energy, are derived to form a complete set of formulas for the total mechanical energy.

\section{Acknowledgements}

This study was supported by the Research Grants from Institute of Crustal Dynamics, China Earthquake Administration (No. ZDJ2017-10). I thank Dr. Kelin Wang, Prof. Jin Ma, Prof. Yuntai Chen, Prof. Wenke Sun, Prof. Yongge Wan, Prof. Jieyuan Ning, Prof. Xiangchu Yin, and all the reviewers who gave helpful comments to improve this new theory.

\section{Conflicts of Interest}

The author declares no conflicts of interest regarding the publication of this paper.

\section{References}

[1] Timoshenko, S. and Goodier, J.N. (1951) Theory of Elasticity. 2nd Edition, McGraw-Hill, New York.

[2] Bullen, K.E. (1963) An Introduction to the Theory of Seismology. 3rd Edition, Cambridge Univ. Press, Cambridge.

[3] Aki, K. and Richards, P.G. (1980) Quantitative Seismology, Theory and Methods. Vol. I, W.H. Freeman and Co., San Francisco.

[4] Lee, W.H.K., Celebi, M., Todorovska, M.I. and Igel, H. (2009) Introduction to the 
Special Issue on Rotational Seismology and Engineering Applications. Bulletin of the Seismological Society of America, 99, 945-957.

https://doi.org/10.1785/0120080344

[5] Lee, W.H.K., Igel, H. and Trifunac, M.D. (2009) Recent Advances in Rotational Seismology. Seismological Research Letters, 80, 479-490.

https://doi.org/10.1785/gssrl.80.3.479

[6] Teisseyre, R.M. (2011) Why Rotation Seismology: Confrontation between Classic and Asymmetric Theories. Bulletin of the Seismological Society of America, 101, 1683-1691. https://doi.org/10.1785/0120100078

[7] Teisseyre, R. (2009) Tutorial on New Development in Physics of Rotation Motions. Bulletin of the Seismological Society of America, 99, 1028-1039.

https://doi.org/10.1785/0120080089

[8] Trifunac, M.D. (1979) A Note on Surface Strains Associated with Incident Body Waves. Bulletin of the European Association of Earthquake Engineering, 5, 85-95.

[9] Nigbor, R. (1994) Six Degree of Freedom Ground Motion Measurement. Bulletin of the Seismological Society of America, 84, 1665-1669.

[10] Spudich, P., Steck, L.K., Hellweg, M., Fletcher, J.B. and Baker, L.M. (1995) Transient Stresses at Parkfield, California, Produced by the M7.4 Landers Earthquake of June 28, 1992: Observations from the UPSAR Dense Seismograph Array. Journal of Geophysical Research, 100, 675-690. https://doi.org/10.1029/94JB02477

[11] Takeo, M. (1998) Ground Rotational Motions Recorded in Near-Source Region. Geophysical Research Letters, 25, 789-792. https://doi.org/10.1029/98GL00511

[12] Igel, H., Schreiber, U., Flaws, A., Schuberth, B., Velikoseltsev, A. and Cochard, A. (2005) Rotational Motions Induced by the M8.1 Tokachi-Oki Earthquake, September 25, 2003. Geophysical Research Letters, 32, L08309. https://doi.org/10.1029/2004GL022336

[13] Cosserat, E. and Cosserat, F. (1909) Théorie des Corps Déformables. Hermann, Paris.

[14] Eringen, A. (1999) Microcontinuum Field Theories, I. Foundation and Solids. Springer, New York. https://doi.org/10.1007/978-1-4612-0555-5

[15] Malvern, L.E. (1969) Introduction to the Mechanics of a Continuous Medium. Prentice-Hall, Upper Saddle River.

[16] Toupin, R. (1964) Theories of Elasticity with Couple Stress. Archive for Rational Mechanics and Analysis, 17, 85-112. https://doi.org/10.1007/BF00253050

[17] Eringen, A. (1966) Linear Theory of Micropolar Elasticity. Journal of Mathematics and Mechanics, 15, 909-923. https://doi.org/10.1512/iumj.1966.15.15060

[18] Cowin, S. (1970) Stress Functions for Cosserat Elasticity. International Journal of Solids and Structures, 6, 389-398. https://doi.org/10.1016/0020-7683(70)90091-0

[19] Nowacki, W. (1986) Theory of Asymmetric Elasticity. PWN and Pergamon Press, Warszawa, Oxford.

[20] Dyszlewicz, J. (2004) Micropolar Theory of Elasticity. Springer, Berlin. https://doi.org/10.1007/978-3-540-45286-7

[21] Pujol, J. (2009) Tutorial on Rotations in the Theories of Finite Deformation and Micropolar (Cosserat) Elasticity. Bulletin of the Seismological Society of America, 99, 1011-1027. https://doi.org/10.1785/0120080161

[22] Ieşan, D. (1981) Some Applications of Micropolar Mechanics to Earthquake Problems. International Journal of Engineering Science, 19, 855-864. 
https://doi.org/10.1016/0020-7225(81)90119-1

[23] Stein, S. and Wysession, M. (2003) An Introduction to Seismology, Earthquake, and Earth Structure. Blackwell Publishing, Oxford.

[24] Aris, R. (1962) Vectors, Tensors, and the Basic Equations of Fluid Mechanics. Dover, Mineola.

[25] Fung, Y.C. (1965) Foundation of Solid Mechanics. Prentice-Hall, Englewood Cliffs.

[26] Muskhelishvili, N.I. (1975) Some Basic Problems of the Mathematical Theory of Elasticity. Noordhoff Intern. Publ., Leyden.

[27] Slaughter, W.S. (2003) The Linearized Theory of Elasticity. Birkhauser, Basel. https://doi.org/10.1007/978-1-4612-0093-2

[28] Richter, C.F. (1958) Elementary Seismology. W. H. Freeman, San Francisco.

[29] Zisman, W.A. (1933) A Comparison of the Statically and Dynamically Determined Elastic Constants of Rocks. Proceedings of the National Academy of Sciences of the United States of America, 19, 680-686. https://doi.org/10.1073/pnas.19.7.680

[30] Lama, R.D. and Vutukuri, V.S. (1978) Handbook on Mechanical Properties of Rock, Vol. II. Trans Tech Publications, Clausthal. 


\section{Appendix}

In the formula (39), the scalar potential satisfies

$$
\nabla \times(\nabla \phi)=0
$$

and the vector potential satisfies both

$$
\nabla \cdot(\nabla \times \psi)=0
$$

and

$$
\nabla \cdot \boldsymbol{\psi}=0
$$

Plugging (39) into (33) we obtain

$$
\rho \frac{\partial^{2}}{\partial t^{2}}(\nabla \phi+\nabla \times \psi)=\left(\lambda+\mu_{2}\right) \nabla\left(\nabla^{2} \phi\right)+\mu_{1} \nabla^{2}(\nabla \phi+\nabla \times \psi) .
$$

where (A2) is used. According to the general relation (24), there is

$$
\nabla^{2}(\nabla \phi+\nabla \times \boldsymbol{\psi})=\nabla\left(\nabla^{2} \phi\right)+\nabla \times\left(\nabla^{2} \boldsymbol{\psi}\right)
$$

where (A1)-(A3) are used, so we have

$$
\rho \frac{\partial^{2}}{\partial t^{2}}(\nabla \phi+\nabla \times \psi)=\left(\lambda+\mu_{1}+\mu_{2}\right) \nabla\left(\nabla^{2} \phi\right)+\mu_{1} \nabla \times\left(\nabla^{2} \psi\right) .
$$

(A6) can be rewritten as

$$
\nabla\left[\rho \frac{\partial^{2} \phi}{\partial t^{2}}-\left(\lambda+\mu_{1}+\mu_{2}\right) \nabla^{2} \phi\right]=-\nabla \times\left(\rho \frac{\partial^{2} \psi}{\partial t^{2}}-\mu_{1} \nabla^{2} \psi\right),
$$

where the scalar and vector potentials are separated each on one side of the equation, respectively. The solution to Equation (A7) can be given by assuming both expressions on each side are equal to zero (e.g., Stein \& Wysession, 2003). Thus, we obtain the wave equation for the scalar potential

$$
\frac{\partial^{2} \phi}{\partial t^{2}}=\frac{\lambda+\mu_{1}+\mu_{2}}{\rho} \nabla^{2} \phi
$$

with the irrotational P-wave velocity (36). Meanwhile, the wave equation for the vector potential is given as

$$
\frac{\partial^{2} \psi}{\partial t^{2}}=\frac{\mu_{1}}{\rho} \nabla^{2} \psi
$$

with the equivoluminal S-wave velocity (38).

\section{Nomenclature}

$\begin{array}{ll}t & \text { time } \\ x_{i}, \quad \boldsymbol{x} & \text { position vector } \\ \boldsymbol{r} & \text { distance vector } \\ u_{i}, \boldsymbol{u} & \text { displacement vector } \\ v_{i}, \boldsymbol{v} & \text { velocity vector } \\ n_{i} & \text { normal vector } \\ t_{i}, \boldsymbol{t} & \text { traction vector }\end{array}$




$\begin{array}{ll}\sigma_{i j} & \text { symmetric stress tensor } \\ \varepsilon_{i j} & \text { symmetric strain tensor } \\ p_{i j} & \text { asymmetric stress tensor } \\ q_{i j} & \text { asymmetric strain tensor } \\ \omega_{i j} & \text { rotation tensor } \\ \boldsymbol{\omega} & \text { rotation vector } \\ \eta_{i j} & \text { stress torque tensor } \\ \eta & \text { stress torque vector } \\ \theta & \text { dilatation } \\ s_{i}, \quad \boldsymbol{s} & \text { spin vector } \\ \lambda, \quad \mu & \text { Lamé's constants } \\ \mu_{1} & \text { parallel shear modulus } \\ \mu_{2} & \text { perpendicular shear modulus } \\ E & \text { Young's modulus } \\ v & \text { Poisson's ratio } \\ c & \text { general elastic modulus } \\ \rho & \text { density } \\ \alpha & \text { P-wave velocity } \\ \beta & \text { S-wave velocity } \\ U & \text { energy } \\ \phi(x, t) & \text { scalar potential } \\ \delta_{i j} & \text { vector potential } \\ e_{i j k} & \text { kronecker delta symbol } \\ \boldsymbol{b} & \text { bermutation symbol } \\ c_{i}, \quad \boldsymbol{c} & \text { body force vector } \\ & \end{array}$

